\title{
A Study Protocol to Assess the Determinants of Glycaemic Control, Complications and Health Related Quality of Life for People with Type 2 Diabetes in Saudi Arabia
}

Mohammed J Alramadan ${ }^{1}$, Afsana Afroz ${ }^{1}$, Mohammed Ali Batais ${ }^{2}$, Turky H Almigbal ${ }^{2}$, Hassan Ahmad Alhamrani ${ }^{3}$, Ahmed Albaloshi $^{4}$, Fatimah A Alramadan ${ }^{3}$, Dianna J Magliano ${ }^{5}$ and Baki Billah ${ }^{* *}$

${ }^{1}$ Department of Epidemiology and Preventive Medicine, Monash University, Melbourne, Australia

${ }^{2}$ College of Medicine, King Saud University, Riyadh, Saudi Arabia

${ }^{3}$ Diabetes Centre, Directorate of Health Affair, Hofuf, Saudi Arabia

${ }^{4}$ Diabetes Centre, Directorate of Health Affair, Jeddah, Saudi Arabia

${ }^{5}$ Baker Heart and Diabetes Institute, Melbourne, Victoria, Australia

\begin{abstract}
Background: The prevalence of type 2 diabetes mellitus is high in Saudi Arabia, and a large proportion of those affected by the disease are not controlling their blood sugar, which exposes them to diabetes complications. The aim of this study is to evaluate factors associated with poor glycaemic control, complications and poor quality of life among people with type 2 diabetes in Saudi Arabia.

Methodology: Using a cross-sectional study design, 1082 participants with type 2 diabetes attending diabetes centres in Riyadh, Jeddah and Hofuf will be recruited in the study. They will be interviewed to complete a pre-tested electronic questionnaire. The questionnaire collects information related to socio-demographics, medical history, lifestyle, family support, utilisation of healthcare services, anxiety, depression, quality of life, cognitive function, independence in daily living activity, neuropathy, anthropometric measures, up-to-date documented lab test results and current medication. Data will be summarised and presented as mean \pm standard deviation (or median and percentiles) for numerical data and frequency and percentage for categorical data. T-test, ANOVA and chi-square tests will be used to explore associations between risk factors and outcomes. Any association will be evaluated further using regression analysis.

Discussion: Knowledge of the risk factors pertaining to poor glycaemic control, diabetes complications and poor quality of life for people with type 2 diabetes is crucial. This knowledge will assist healthcare providers to identify and provide more intensive care plans to those who need it, as well as guide the development of new strategies to improve management of the disease. This will improve the health of people with type 2 diabetes and lower their risk of complications, and reduce the burden of this highly prevalent disease on families and the community.
\end{abstract}

Keywords: Type 2 diabetes; Saudi Arabia; Glycaemic control; Complication; Quality of life

\section{Introduction}

Diabetes mellitus is one of the most common chronic noncommunicable diseases [1,2]. An estimated 415 million adults had diabetes worldwide in 2015 and this number is expected to rise to 642 million by 2040 [3]. In 2015, approximately five million individuals between the age of 20 and 79 years died because of diabetes [3]. Furthermore, roughly $14.5 \%$ of global all-cause mortality among adults is attributed to diabetes and half of these deaths occur among those aged below 60 [3].

Similar to other countries in the Middle East, modernisation has taken its toll on the population of Saudi Arabia. The vast lifestyle changes of Saudis over the last few decades, accompanied by rising rates of obesity, have led to a rapid and progressive increase in the prevalence of diabetes. A recent study showed that $13.4 \%$ of Saudis aged 15 years and above have diabetes, which is significantly higher than both the global prevalence (8.8\%) and the prevalence in the Middle East (10.7\%) $[3,4]$.

The control of blood glucose levels is the cornerstone of diabetes management. Studies have shown that there is a strong association between an elevated blood glucose level and the risk of diabetes-related complications and mortality for people with diabetes $[5,6]$. Of particular concern is that between $50 \%$ and $70 \%$ of people with type 2 diabetes mellitus (T2DM) in Saudi Arabia have uncontrolled blood glucose levels [7-9], and the prevalence of diabetes-related complications among them is high [10-13].

A few studies that have examined factors affecting glycaemic control for people with T2DM in Saudi Arabia have indicated poor control to be associated with increasing age, insulin use, smoking, lower levels of physical activity, poor diabetes self-care behaviour, low adherence to medicine, anxiety and depression [7-9]. A number of other factors that may have an impact on glycaemic control, diabetes complications and quality of life-such as the duration of diabetes, family support, cognitive function and lifestyle factors (diet and physical activity)-were not investigated adequately in existing studies from Saudi Arabia [14-16].

Furthermore, it is well-established that haemoglobin A1c (HbAlc) is a more accurate measure of blood sugar control, compared to fasting

*Corresponding author: Baki Billah, Department of Epidemiology and Preventive Medicine, Monash University, Melbourne, Australia, Tel: +61399030160; Fax: +61399030556; E-mail: baki.billah@monash.edu

Received May 12, 2016; Accepted June 08, 2017; Published June 12, 2017

Citation: Alramadan MJ, Afroz A, Batais MA, Almigbal TH, Alhamrani HA, et al. (2017) A Study Protocol to Assess the Determinants of Glycaemic Control, Complications and Health Related Quality of Life for People with Type 2 Diabetes in Saudi Arabia. J Health Educ Res Dev 5: 219. doi: 10.4172/2380-5439.1000219

Copyright: @ 2017 Alramadan MJ, et al. This is an open-access article distributed under the terms of the Creative Commons Attribution License, which permits unrestricted use, distribution, and reproduction in any medium, provided the original author and source are credited. 
Citation: Alramadan MJ, Afroz A, Batais MA, Almigbal TH, Alhamrani HA, et al. (2017) A Study Protocol to Assess the Determinants of Glycaemic Control, Complications and Health Related Quality of Life for People with Type 2 Diabetes in Saudi Arabia. J Health Educ Res Dev 5: 219. doi: $10.4172 / 2380-5439.1000219$

Page 2 of 6

and random blood sugar measurements, because $\mathrm{HbAlc}$ reflects the level of glycaemic control over several weeks [17]. However, some of the studies from Saudi Arabia used random blood sugar for determining the level of control, which was a limitation to these studies. In addition, the majority of studies from Saudi Arabia involved a single centre, or were hospital-based $[8-10,13,18-26]$ and may not accurately represent the large and diverse population of the country.

To address the above gaps, a multi-centre study is required which will comprehensively investigate the effect of all possible risk factors. Thus, the aim of this proposed study is to conduct a multi-centre study in Saudi Arabia to explore the effect of lifestyle factors, family support and cognitive impairment, as well as other risk factors for glycaemic control, diabetes complications and quality of life for people with T2DM mellitus (T2DM). The knowledge of these risk factors will help healthcare providers, the individuals with the disease, and society as a whole. Understanding the burden of the associated risk factors is also important for public health policymakers who develop healthcare priorities that yield the greatest benefits.

\section{Objectives}

This study has three main objectives. Firstly, to evaluate the determinants of poor glycaemic control among people with T2DM in Saudi Arabia. Secondly, to investigate the prevalence of coronary artery disease, neuropathy, nephropathy and cognitive impairment, as well as their associated factors among people with T2DM. Thirdly, to examine factors associated with poor health-related quality of life for people with T2DM. Furthermore, secondary objectives of this study is to examine factors associated with poor control of blood pressure as well dyslipidaemia among people with T2DM.

\section{Methodology}

\section{Ethical approval}

Both the Monash University Human Research Ethics Committee and the Research Ethics Committee of the Ministry of Health in Saudi Arabia approved this project. The approval of the College of Medicine Institutional Review Board at Kind Saud University was also obtained.

\section{Study design}

A cross-sectional study will be conducted to address the research questions. Cross-sectional study design has the advantage of being inexpensive and less time-consuming compared to other epidemiological study designs. Moreover, in using this study design, the prevalence of outcomes such as poor glycaemic control and complications, as well as the prevalence of risk factors, can be measured.

\section{Study population}

The study population will consist of people with T2DM in Saudi Arabia attending diabetes centres in the cities of Hofuf, Riyadh and Jeddah (Figure 1). These cities are among the top most populated in the country and their diabetic centres serve a mixture of people who come from urban, as well as rural settings. Inclusion criteria include confirmed diagnosis of T2DM, age 18 years and above and duration of diabetes of one year and more. Participants will be excluded if they have other types of diabetes (type 1 or gestational), or if there is no HbAlc test results in their medical records for the past 6 months. Pregnant women will also be excluded.

\section{Sample size}

A total of 1082 subjects with T2DM will be recruited for the study which was calculated based on $90 \%$ power, $5 \%$ significance level and a margin of error of $2.5 \%$ for prevalence of glycaemic control. A back calculation of power shows that a sample size of 1082 participants will maintain a power of $90 \%$ or above for all other primary and secondary objectives of this study.

\section{Recruitment}

A systematic random-sampling method will be used to recruit participants. The number of participants recruited each day is expected to be 10. Every day during the data-collection period, the data collectors will begin by randomly selecting a participant with T2DM from the first $\mathrm{K}$ participants attending the diabetes centre and invite them to participate. The value of $\mathrm{K}$ will depend on the number of people attending the centre every day, which varies between centres. Following on, every K-th patient will be approached. If the K-th person declines or does not have T2DM, the next person will be invited. The recruitment will be continued for a period of six months or until data have been collected from 1082 patients, whichever comes first.

\section{Informing participants about the study and obtaining the consent}

Data collectors will fully inform each of the participants about the purpose of the study, how the data will be collected and how the collected information will be used while maintaining participants confidentiality. Then, participants will be given an explanatory statement and allowed some time to read and ask questions. Upon their agreement to participate, they will be required to read and sign a consent form.

\section{Data collection instrument}

A pre-tested questionnaire will be used to collect data. The original English version of the questionnaire has been translated into Arabic. The Arabic version was then translated into English to check that the Arabic and the original English versions have exactly the same meanings. Prior to collecting the data, data collectors will be trained by the primary investigator on how to approach participants, inform participants about the study, fill in the electronic questionnaire and take the anthropometric measurements.

Research Electronic Data Capture (REDCap) will be used to collect and manage the data [27]. REDCap is a secure web-based application

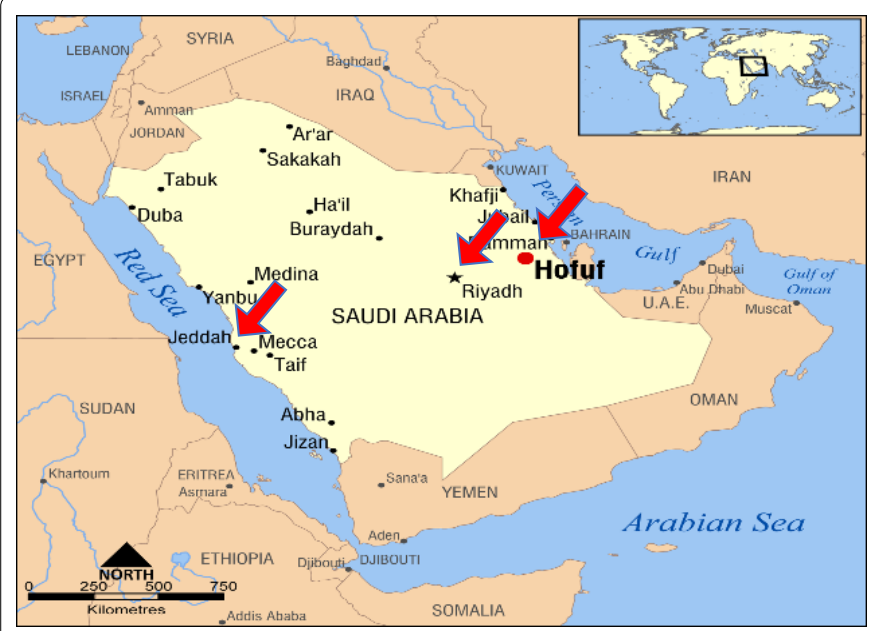

Figure 1: Map of Saudi Arabia showing the main cities and the cities where the research will be conducted (red arrows). 
Citation: Alramadan MJ, Afroz A, Batais MA, Almigbal TH, Alhamrani HA, et al. (2017) A Study Protocol to Assess the Determinants of Glycaemic Control, Complications and Health Related Quality of Life for People with Type 2 Diabetes in Saudi Arabia. J Health Educ Res Dev 5: 219. doi: $10.4172 / 2380-5439.1000219$

Page 3 of 6

for constructing electronic surveys and collecting data for research studies. It provides a user-friendly interface with validated data entry, audit trails for tracking data manipulation, and an automated export procedure for seamless data downloads to common statistical software packages.

Using a standard questionnaire, the following information will be collected for all participants:

- Diabetes centre and data collector details.

- Participant's socio-demographics data, including name, age, gender, marital status, education, nationality, work status and household income.

- Medical history: duration of diabetes, place of follow-up, frequency of follow-up, diabetes treatment, self-monitoring blood glucose, hypoglycaemic events, medical history of other diseases, diabetes complications and medication adherence (Morisky Medication Adherence questionnaire).

- Family support for diabetes, utilisation of healthcare services, knowledge of HbAlc.

- Lifestyle data including smoking status, dietary habits (UK diabetes and diet questionnaire at: https://sps.onlinesurveys. ac.uk/the-uk-diabetes-and-diet-questionnaire-ukddq) and physical activity (WHO STEPS questionnaire for diet at: http:// www.who.int/chp/steps/STEPS_Instrument_v2.1.pdf).

- Psychological aspect data that include depression (the Patient Health Questionnaire-2 (PHQ-2)) [28] and anxiety (Generalized Anxiety Disorder Scale (GAD-2)) [29].

- Patient's quality of life (EQ-5D-5L) [30] and independence regarding activities in daily life (Katz Index) [31].

- Rowland Universal Dementia Assessment Scale (RUDAS) [32].

- Neuropathy screening tool (the Michigan Neuropathy Screening Instrument [33]).

- Anthropometrics: height, weight, blood pressure and waist and hip circumference.

- Materials and equipment: digital scale, measuring tape, and digital automatic blood pressure monitor

- Height: height will be measured for all participants using a portable stadiometer. Standing height is measured with the subject in bare feet, back-square against the wall and eyes looking straight ahead. A set square resting on the scalp and a tape measurement from the wall/bed is used to measure height to the nearest $0.5 \mathrm{~cm}$. This will be done twice and if the measurement varies more than $2 \mathrm{~cm}$, a third measurement will be taken. A stool will be used where necessary.

- Weight: participant will be instructed to remove their shoes and outer layers of clothing (such as jackets or jumpers). Weight will be recorded to the nearest $0.1 \mathrm{~kg}$.

- Waist circumference: will be measured against thin clothing (for cultural reasons), on exhalation, midway between the lower rib margin and the anterior superior iliac spine (hip bone) or narrowest abdominal point. Subject should be relaxed with arms held loosely at sides. The tape measure must be kept horizontal for standing measurement. This will be done twice and if the measurement varies by more than $2 \mathrm{~cm}$, a third measurement will be taken. The waist circumference will be recorded to the nearest $0.5 \mathrm{~cm}$.

- Hip circumference: measure at the widest circumference around the hip bones, so that the tape passes over the greatest protrusion of the gluteal muscles. The tape measure must be kept horizontal for standing measurement. This will be done twice and if the measurement varies more than $2 \mathrm{~cm}$, a third measurement will be taken. The hip circumference will be recorded to the nearest $0.5 \mathrm{~cm}$.

- Blood pressure: participants should be seated for at least five minutes, legs uncrossed, feet flat on the floor and any excess clothing items that may interfere with measurement must be removed. Three systolic and diastolic blood pressure readings will be taken using an automated blood pressure monitor machine.

- Information from patients' medical records, including the three most recent blood pressure measures, fasting blood sugar measures, $\mathrm{HbA1c}$, serum creatinine, albumin/creatinine ratio, eGFR, lipid profile, current prescribed medications and documented diagnosis of hypertension, coronary artery disease and stroke.

\section{Data management and analysis}

During the data-collection period, the data will be saved in the secure REDCap web-based application hosted by Monash University. The application is accessible only by the research team. When the data collection is completed the data will be exported to the IBM SPSS statistical package and will be saved on the secure School of Public Health and Preventive Medicine at Monash University allocated network storage (Monash (S:) drive). Participants' names will be removed from the database and each participant will be identified by a numeric code generated by REDCap. The database containing all information will be saved in a separate secure electronic folder, which will not be used for data analysis. Only the research team will have access to the identified and de-identified electronic databases.

Data will be summarised and presented as either mean \pm standard deviation or median and percentiles for numerical data and frequency, and percentage for categorical data. Depending on the type of data, t-test, ANOVA, nonparametric tests or chi-square tests will be used to examine for associations between risk factors and outcomes. Any association will be further evaluated using simple and multiple logistic regression analysis.

\section{Outcomes assessment}

Definitions of the main study outcomes that will be considered:

- Glycaemic control: the proportion of the glycosylated haemoglobin $(\mathrm{HbAlc})$ measured as a percentage and categorised as good control $(\mathrm{HbAlc}<7.0 \%)$, reasonable control (HbAlc 7\%-8\%) and poor control (HbAlc $>8.0 \%)$.

- Nephropathy: documented eGFR (calculated by the CKD EPI formula) below $90 \mathrm{ml} / \mathrm{min}$ (categorised as mild renal impairment (60-89 $\mathrm{ml} / \mathrm{min})$, moderate renal impairment (30$59 \mathrm{ml} / \mathrm{min})$, severe renal impairment $(15-29 \mathrm{ml} / \mathrm{min})$ and renal failure $(<15 \mathrm{ml} / \mathrm{min})$.

- Coronary artery disease: documented diagnosis of coronary artery disease (CAD). 
Citation: Alramadan MJ, Afroz A, Batais MA, Almigbal TH, Alhamrani HA, et al. (2017) A Study Protocol to Assess the Determinants of Glycaemic Control, Complications and Health Related Quality of Life for People with Type 2 Diabetes in Saudi Arabia. J Health Educ Res Dev 5: 219. doi: $10.4172 / 2380-5439.1000219$

Page 4 of 6

- Neuropathy: a score of 7 or more using the Michigan Neuropathy Screening Instrument.

- Quality of life: EQ-5D-5L health states scores will be converted into a single index value between 0 and 1 , and the quality of life will be categorised as Good quality of life (0.67-1.00), fair quality of life (0.34-0.66) and poor quality of life $(\leq 0.33)$.

- Hypertension: either a documented diagnosis of hypertension, on antihypertension medications or three previous high blood pressure readings (systolic $\geq 140$ and diastolic $\geq 90$ ). Persons with poor blood pressure control are defined as having hypertension and their current systolic blood pressure as $\geq 140$ $\mathrm{mm} \mathrm{Hg}$ or diastolic $\geq 90 \mathrm{~mm} \mathrm{Hg}$.

- Dyslipidaemia: Documented total cholesterol $>4.0 \mathrm{mmol} / \mathrm{L}$, low density lipoproteins (LDL) $>2.0 \mathrm{mmol} / \mathrm{L}$, high density lipoprotein $(\mathrm{HDL})<1.0 \mathrm{mmol} / \mathrm{L}$, triglycerides $>2.0 \mathrm{mmol} / \mathrm{L}$ or taking lipid lowering medication.

- Impaired cognitive function is defined as a score of $\leq 22$ in the Rowland Universal Dementia Assessment Scale (RUDAS).

\section{Pilot Study}

The questionnaire was piloted on 29 participants attending diabetes centre in Hofuf over a period of two weeks. The sociodemographic characteristics of the participants in the pilot study are presented in Table 1. Mean age was 55.7 ( \pm 11.6 ) years. Approximately $38 \%$ of participants were female and the majority of participants were married (93.1\%). Roughly a quarter $(24.1 \%)$ of participants was illiterate and $41.4 \%$ had only achieved primary school. The majority of participants $(82.8 \%)$ reside in Hofuf city, while the rest lived in remote villages. With regard to work status, $17.2 \%$ worked and $34.5 \%$ were homemakers (house-wives). The rest of the participants were either retired (27.6\%) or not working (20.7\%). A large proportion of participants (41.4\%) had a total household income between 3000 and 6000 Saudi Riyals.

A summary of some disease characteristics and main study outcomes is presented in Table 2. Participants had a mean duration of diabetes of 17.7 ( \pm 10.5 ) years. Mean HbAlc was 9.6\% ( $\pm 2.0 \%)$. The majority of participants $(69.0 \%)$ had poor glycaemic control (HbAlc $>8$ ), while $10.3 \%$ had good glycaemic control (HbAlc $\leq 7 \%)$ and $17.2 \%$ had reasonable control (HbAlc 7.1\%-8.0\%). More than half of the participants (58.6\%) had dyslipidaemia and close to half (48.3\%) had hypertension, while the diagnosis of CAD was documented in $31.0 \%$ of participants. Using the Michigan Neuropathy Screening Instrument, $31.0 \%$ of participants had a score $(\geq 7)$, suggestive of neuropathy. eGFR calculated from most recent serum creatinine showed that $55.2 \%$ of participants had some renal impairment. Of those, $27.6 \%$ had mild impairment, $24.1 \%$ had moderate impairment, and $3.4 \%$ had severe impairment. Cognitive function was impaired in about half (51.7\%) of participants using the Six-item Cognitive Impairment Test (6CIT) [34]. The quality of life was fair for $34.5 \%$ and poor for $3.4 \%$ of participants, while the majority (62.1\%) had good quality of life. There were some missing values in some important investigations including $\mathrm{HbAlc}$, cholesterol and triglycerides. The range of missing values was between $3.4 \%$ for $\mathrm{HbAlc}$ and $5.0 \%$ for triglycerides.

The questionnaire was found to be acceptable by most of the participants and practical by data collectors. However, the average time required to complete data collection for one participant was more than one hour. In order to ensure the collection of data from the planned number of participants, the length of the questionnaire was reduced after the completion of the pilot study. The STOP-BANG Sleep Apnea and the chest pain (Rose) questionnaires were removed $[35,36]$. Questions related to the duration of complications reported by participants were also removed.

Based on our findings in the pilot study that a relatively large proportion of people were illiterate, a decision was made to use the Rowland Universal Dementia Assessment Scale (RUDAS) instead of the Six-item Cognitive Impairment Test (6-CIT) to evaluate cognitive function. RUDAS is a multicultural cognitive assessment tool that is not affected by literacy level, and has been validated among Arabicspeaking people [32,37]. Approval to use the amended questionnaire was obtained from all ethical committees that have approved this project.

\section{Discussion}

This project is a comprehensive multi-centre study that will improve our understanding of the factors associated with poor control of blood glucose level, the factors that increase risk of diabetes complications and the factors associated with poor quality of life among people with T2DM in Saudi Arabia. The anticipated valuable information that will be obtained from this study will help healthcare providers to identify people with diabetes who are at risk of poor control and complications,

\begin{tabular}{|c|c|c|c|}
\hline Variable & Descriptive statistics & Variable & Descriptive statistics \\
\hline Age in years (mean $\pm S D$ ) & $55.7 \pm 11.6$ & \multirow{3}{*}{$\begin{array}{c}\text { Work status \% } \\
\text { Working } \\
\text { Not working (able to work) } \\
\text { Not working (unable to work) } \\
\text { Homemaker (house-wife) } \\
\text { Retired }\end{array}$} & \multirow{3}{*}{$\begin{array}{c}5(17.2 \%) \\
2(6.9 \%) \\
4(13.8 \%) \\
10(34.5 \%) \\
8(27.6 \%)\end{array}$} \\
\hline $\begin{array}{l}\text { Gender \% } \\
\text { Female } \\
\text { Male }\end{array}$ & $\begin{array}{l}11(37.9 \%) \\
18(62.1 \%)\end{array}$ & & \\
\hline $\begin{array}{c}\text { Marital status \% } \\
\text { Married }\end{array}$ & \multirow{2}{*}{$\begin{array}{c}27(93.1 \%) \\
1(3.4 \%) \\
1(3.4 \%)\end{array}$} & & \\
\hline $\begin{array}{c}\text { Divorced (separated) } \\
\text { Widowed }\end{array}$ & & \multirow{3}{*}{$\begin{array}{c}\text { Total household monthly income } \% \\
\leq 3000 \text { Saudi Riyals } \\
3000-6000 \text { Saudi Riyals } \\
6001-9000 \text { Saudi Riyals } \\
9001-12000 \text { Saudi Riyals } \\
\geq 12001 \text { Saudi Riyals }\end{array}$} & \multirow{3}{*}{$\begin{array}{c}3(10.3 \%) \\
12(41.4 \%) \\
4(13.8 \%) \\
7(24.1 \%) \\
3(10.3 \%)\end{array}$} \\
\hline $\begin{array}{c}\text { Highest education level achieved \% } \\
\text { None (illiterate) } \\
\text { Primary school } \\
\text { Intermediate school } \\
\text { Tertiary school } \\
\text { University degree }\end{array}$ & $\begin{array}{c}7(24.1 \%) \\
12(41.4 \%) \\
1(3.4 \%) \\
8(27.6 \%) \\
1(3.4 \%)\end{array}$ & & \\
\hline $\begin{array}{l}\text { Home location \% } \\
\text { Inside the city } \\
\text { Remote village }\end{array}$ & $\begin{array}{l}24(82.8 \%) \\
5(17.2 \%)\end{array}$ & & \\
\hline
\end{tabular}

Table 1: Pilot study participants' sociodemographic characteristics. 
Citation: Alramadan MJ, Afroz A, Batais MA, Almigbal TH, Alhamrani HA, et al. (2017) A Study Protocol to Assess the Determinants of Glycaemic Control, Complications and Health Related Quality of Life for People with Type 2 Diabetes in Saudi Arabia. J Health Educ Res Dev 5: 219. doi: $10.4172 / 2380-5439.1000219$

Page 5 of 6

\begin{tabular}{|c|c|c|c|}
\hline Variable & Descriptive statistics & Variable & Descriptive statistics \\
\hline Diabetes duration in years (mean $\pm S D$ ) & $17.7 \pm 10.5$ & \multirow{2}{*}{$\begin{array}{l}\text { Hypertension \% } \\
\text { No } \\
\text { Yes }\end{array}$} & \multirow{2}{*}{$\begin{array}{l}15(51.7 \%) \\
14(48.3 \%)\end{array}$} \\
\hline HbA1c \% (mean \pm SD) & $9.6 \pm 2.0$ & & \\
\hline $\begin{array}{c}\text { Glycaemic control \% } \\
\text { Good } \\
\text { Reasonable } \\
\text { Poor }\end{array}$ & $\begin{array}{c}3(10.3 \%) \\
5(17.2 \%) \\
20(69.0 \%)\end{array}$ & $\begin{array}{l}\text { CAD \% } \\
\text { No } \\
\text { Yes }\end{array}$ & $\begin{array}{c}20(69.0 \%) \\
9(31.0 \%)\end{array}$ \\
\hline $\begin{array}{l}\text { Quality of life \% } \\
\text { Good } \\
\text { Fair } \\
\text { Poor }\end{array}$ & $\begin{array}{c}18(62.1 \%) \\
10(34.5 \%) \\
1(3.4 \%)\end{array}$ & $\begin{array}{l}\text { Neuropathy \% } \\
\text { No } \\
\text { Yes }\end{array}$ & $\begin{array}{c}20(69.0 \%) \\
9(31.0 \%)\end{array}$ \\
\hline $\begin{array}{c}\text { Cognitive impairment } \% \\
\text { No } \\
\text { Yes }\end{array}$ & $\begin{array}{l}14(48.3 \%) \\
15(51.7 \%)\end{array}$ & \multirow{2}{*}{$\begin{array}{c}\text { Nephropathy \% } \\
\text { No } \\
\text { Mild } \\
\text { Moderate } \\
\text { Severe }\end{array}$} & \multirow{2}{*}{$\begin{array}{c}13(44.8 \%) \\
8(27.6 \%) \\
7(24.1 \%) \\
1(3.4 \%)\end{array}$} \\
\hline $\begin{array}{c}\text { Dyslipidaemia \% } \\
\text { No } \\
\text { Yes }\end{array}$ & $\begin{array}{l}12(41.4 \%) \\
17(58.6 \%)\end{array}$ & & \\
\hline
\end{tabular}

Table 2: Characteristics related to diabetes, complications and health related quality of life.

and provide them with more intensive care plans. It will also identify priority issues that will guide the development and implementation of new national strategies to promote the health of people with T2DM in Saudi Arabia.

The strength of this study lies on the relatively large number of participants and the recruitment from three highly populated cities in three different regions of Saudi Arabia. The evaluation of a wide range of risk factors and outcomes using validated tools also provide strength to this study. Since this study is cross-sectional, we will be assessing association; no causal relation can be inferred. Observational study designs are also prone to bias. Nevertheless, this study will not only provide valuable information that will be used by healthcare providers and health policy makers, but will also generate hypotheses that will guide future advance research projects in the field of diabetes in Saudi Arabia, in the Middle East region and globally.

\section{Competing Interests}

All authors declare that they have no competing interests.

\section{Author Contributions}

All authors were involved in the conception and design of the study. MJA and AA reviewed the literature and MJA drafted the manuscript. All authors critically reviewed the manuscript and approved the final version.

\section{References}

1. Shaw JE, Sicree RA, Zimmet PZ (2010) Global estimates of the prevalence of diabetes for 2010 and 2030. Diabetes Research and Clinical Practice 87: 4-14.

2. Whiting DR, Guariguata L, Weil C, Shaw J (2011) IDF diabetes atlas: global estimates of the prevalence of diabetes for 2011 and 2030. Diabetes Research and Clinical Practice 94: 311-321.

3. Cho NH, Whiting D, Forouhi N, Leonor G, Hambleton I, et al. (2015) IDF Diabetes Atlas. 7th edn.

4. El Bcheraoui C, Basulaiman M, Tuffaha M, Daoud F, Robinson M, et al (2014) Status of the diabetes epidemic in the Kingdom of Saudi Arabia, 2013. International Journal of Public Health 59: 1011-1021.
5. Control D, Group CTR (1993) The effect of intensive treatment of diabetes on the development and progression of long-term complications in insulindependent diabetes mellitus. N Engl J Med 329: 977-986.

6. Group UPDS (1998) Intensive blood-glucose control with sulphonylureas or insulin compared with conventional treatment and risk of complications in patients with type 2 diabetes (UKPDS 33). The Lancet 352: 837-853.

7. Al-Nuaim AR, Mirdad S, Al-Rubeaan K, Al-Mazrou Y, Al-Attas O, et al. (1998) Pattern and factors associated with glycemic control of Saudi diabetic patients. Ann Saudi Med 18: 109-112.

8. Al-Hayek AA, Robert AA, Alzaid AA, Nusair HM, Zbaidi NS, et al. (2012) Association between diabetes self-care, medication adherence, anxiety, depression, and glycemic control in type 2 diabetes. Saudi Medical Journal 33: $681-683$

9. Alsulaiman TA, Al-Ajmi HA, Al-Qahtani SM, Fadlallah IM, Nawar NE, et al. (2016) Control of type 2 diabetes in King Abdulaziz Housing City (Iskan) population, Saudi Arabia. Journal of Family and Community Medicine 23: 1.

10. Alwakeel J, Sulimani R, Al-Asaad H, Al-Harbi A, Tarif N, et al. (2008) Diabetes complications in 1952 type 2 diabetes mellitus patients managed in a single institution. Annals of Saudi Medicine 28: 260

11. Al Ghamdi AH, Rabiu M, Hajar S, Yorston D, Kuper H, et al. (2012) Rapid assessment of avoidable blindness and diabetic retinopathy in Taif, Saud Arabia. British Journal of Ophthalmology 96: 1168-1172.

12. Halawa MR, Karawagh A, Zeidan A, Mahmoud DH, Sakr M, et al. (2010) Prevalence of painful diabetic peripheral neuropathy among patients suffering from diabetes mellitus in Saudi Arabia. Current Medical Research and Opinion 26: 337-343.

13. Ahmed AA, Algamdi SA, Alzahrani AM (2015) Surveillance of risk factors for diabetic foot ulceration with particular concern to local practice. Diabetes \& Metabolic Syndrome: Clinical Research \& Reviews 9: 310-315.

14. Sanal T, Nair N, Adhikari P (2011) Factors associated with poor control of type 2 diabetes mellitus: a systematic review and meta-analysis. Journal of Diabetology 3: 1-10.

15. Stopford R, Winkley K, Ismail K (2013) Social support and glycemic control in type 2 diabetes: a systematic review of observational studies. Patient Education and Counseling 93: 549-558 
Citation: Alramadan MJ, Afroz A, Batais MA, Almigbal TH, Alhamrani HA, et al. (2017) A Study Protocol to Assess the Determinants of Glycaemic Control, Complications and Health Related Quality of Life for People with Type 2 Diabetes in Saudi Arabia. J Health Educ Res Dev 5: 219. doi: $10.4172 / 2380-5439.1000219$

Page 6 of 6

16. Awad N, Gagnon M, Messier C (2004) The relationship between impaired glucose tolerance, type 2 diabetes, and cognitive function. Journal of Clinical and Experimental Neuropsychology 26: 1044-1080.

17. Association AD (2016) Standards of medical care in diabetes - 2016. Diabetes Care.

18. Al-Rubeaan K, Al-Hussain F, Youssef AM, Subhani SN, Al-Sharqawi AH, et al. (2016) Ischemic stroke and its risk factors in a registry-based large crosssectional diabetic cohort in a country facing a diabetes epidemic. Journal of Diabetes Research.

19. Al-Rubeaan K, El-Asrar A, Ahmed M, Youssef AM, Subhani SN, et al. (2015) Diabetic retinopathy and its risk factors in a society with a type 2 diabetes epidemic: a Saudi National Diabetes Registry-based study. Acta Ophthalmologica 93: e140-e147.

20. El-Asrar AMA, Al-Rubeaan KA, Al-Amro SA, Kangave D, Moharram OA (1998) Risk factors for diabetic retinopathy among Saudi diabetics. International Ophthalmology 22: 155-161.

21. Wang DD, Bakhotmah BA, Hu FB, Alzahrani HA (2014) Prevalence and correlates of diabetic peripheral neuropathy in a saudi arabic population: a cross-sectional study. PloS ONE 9: e106935.

22. Al-Homrany MA, Abdelmoneim I (2004) Significance of proteinuria in type 2 diabetic patients treated at a primary health care center in Abha City, Saudi Arabia. West African Journal of Medicine 23: 211-214.

23. Al-Rubeaan K, Youssef AM, Subhani SN, Ahmad NA, Al-Sharqawi AH, et al. (2014) Diabetic nephropathy and its risk factors in a society with a type 2 diabetes epidemic: a Saudi National Diabetes Registry-based study. PloS ONE 9: e88956.

24. Al-Shehri A, Taha A, Bahnassy A, Salah M (2008) Health-related quality of life in type 2 diabetic patients. Annals of Saudi Medicine 28: 352

25. Al Hayek AA, Robert AA, Al Saeed A, Alzaid AA, A Sabaan FS (2014) Factors associated with health-related quality of life among Saudi patients with type 2 diabetes mellitus: a cross-sectional survey. Diabetes \& Metabolism Journal 38 220-229.

26. AL-Aboudi IS, Hassali MA, Shafie AA, Hassan A, Alrasheedy AA (2015) A crosssectional assessment of health-related quality of life among type 2 diabetes patients in Riyadh, Saudi Arabia. SAGE Open Medicine.

27. Harris PA, Taylor R, Thielke R, Payne J, Gonzalez N, et al. (2009) Research electronic data capture (REDCap)-a metadata-driven methodology and workflow process for providing translational research informatics support. Journal of Biomedical Informatics 42: 377-381.

28. Kroenke K, Spitzer RL, Williams JB (2003) The Patient Health Questionnaire-2: validity of a two-item depression screener. Medical Care 41: 1284-1292.

29. Skapinakis P (2007) The 2-item Generalized Anxiety Disorder scale had high sensitivity and specificity for detecting GAD in primary care. Evidence Based Medicine 12: 149.

30. Herdman M, Gudex C, Lloyd A, Janssen M, Kind P, et al. (2011) Developmen and preliminary testing of the new five-level version of EQ-5D (EQ-5D-5L). Quality of Life Research 20: 1727-1736

31. Shelkey M, Wallace M (1999) Katz index of independence in activities of daily living. Journal of Gerontological Nursing 25: 8-9.

32. Storey JE, Rowland JT, Conforti DA, Dickson HG (2004) The Rowland universa dementia assessment scale (RUDAS): a multicultural cognitive assessment scale. International Psychogeriatrics 16: 13-31.

33. Moghtaderi A, Bakhshipour A, Rashidi H (2006) Validation of Michigan neuropathy screening instrument for diabetic peripheral neuropathy. Clinical Neurology and Neurosurgery 108: 477-481.

34. Abdel-Aziz K, Larner A (2015) Six-item cognitive impairment test (6CIT) pragmatic diagnostic accuracy study for dementia and $\mathrm{MCl}$. International Psychogeriatrics 27: 991-997.

35. Rose G, McCartney P, Reid D (1977) Self-administration of a questionnaire on chest pain and intermittent claudication. British Journal of Preventive \& Socia Medicine 31: 42-48.

36. Chung F, Subramanyam R, Liao P, Sasaki E, Shapiro C, et al. (2012) High STOP-Bang score indicates a high probability of obstructive sleep apnoea. British Journal of Anaesthesia: aes022.

37. Chaaya M, Phung T, El Asmar K, Atweh S, Ghusn H, et al. Validation of the Arabic Rowland Universal Dementia Assessment Scale (A-RUDAS) in elderly with mild and moderate dementia. Aging \& Mental Health 20: 880-887. 